\title{
Drug discovery from Chinese medicine against neurodegeneration in Alzheimer's and vascular dementia
}

\author{
Yuen-Shan $\mathrm{Ho}^{1 *}$, Kwok-Fai So ${ }^{1,2,3}$ and Raymond Chuen-Chung Chang ${ }^{1,2,3^{*}}$
}

\begin{abstract}
Alzheimer's disease and vascular dementia are two major diseases associated with dementia, which is common among the elderly. While the etiology of dementia is multi-factorial and complex, neurodegeneration may be the major cause of these two diseases. Effective drugs for treating dementia are still to be discovered. Current western pharmacological approaches against neurodegeneration in dementia develop symptom-relieving and diseasemodifying drugs. Current integrative and holistic approaches of Chinese medicine to discovering drugs for neurodegeneration in dementia include (1) single molecules from the herbs, (2) standardized extracts from a single herb, and (3) herbal formula with definite composition. This article not only reviews the concept of dementia in western medicine and Chinese medicine but also evaluates the advantages and disadvantages of these approaches.
\end{abstract}

\section{Introduction}

Alzheimer's disease (AD) and vascular dementia (VaD) are the major forms of dementia. In addition, in the postmortem brains of the late stage of Parkinson's disease/Lewy body disease also find pathological hallmarks of $\mathrm{AD}$ [1]. Senile dementia is the progressive decline of memory and some related cognitive functions in the elderly. The global dementia population is predicted to reach 81.1 million by 2040 [2]. In 2010, the estimated prevalence of senile dementia in China is 6.0 to 7.0 million, accounting for about one-sixth of the global prevalence; the prevalence is expected to increase to 22.5 million by 2040, accounting for one-fourth of the global prevalence by that time [3]. The rapid increase in the number of dementia patients urgently demands effective prevention and treatment. Current approaches to dementia-related neurodegenerative diseases still highly rely on relieving symptoms. As some Chinese medicinal herbs have been used in treating dementia, many researchers are now turning to Chinese medicine for identifying potential neuroprotective agents or diseasemodifying agent. This article reviews the strategy in the

\footnotetext{
* Correspondence: janiceys@hku.hk; rccchang@hku.hk

'Laboratory of Neurodegenerative Diseases, Department of Anatomy, The University of Hong Kong Pokfulam, Hong Kong SAR, China

Full list of author information is available at the end of the article
}

research of Chinese medicine in dementia related-neurodegenerative diseases.

\section{Dementia and medical sciences}

$\mathrm{AD}$ is clinically characterized by the progressive loss of memory, cognitive functions and behavioral changes. The pathogenesis of $\mathrm{AD}$ has been widely studied $[4,5]$, in which beta-amyloid $(A \beta)$ peptide and hyperphosphorylated tau protein as components of extracellular senile plaques and intracellular neurofibrillary tangles, respectively, are believed to be the targets for developing disease-modifying drugs. Current AD treatments are all symptom-relieving agents and heavily rely on the use of acetylcholinesterase (AChE) inhibitors (donepezil, rivastigmine and galantamine). AChE inhibitors slow down the degradation of the neurotransmitter acetylcholine, thereby increasing its bioavailability. Another approved AD treatment aims to reduce glutamate excitotoxicity. Memantine, the only approved drug in this category, acts as a non-competitive $N$-methyl-D-aspartate (NMDA) receptor antagonist to reduce glutamatemediated neurotoxicity [6].

Development and progression of $\mathrm{VaD}$ are associated with a number of risk factors, many of which are related to the pathogenesis of atherosclerosis [7]. Stroke is also a critical factor for $\mathrm{VaD}$; it was reported that $79.5 \%$ of $\mathrm{VaD}$ 
patients had a history of stroke [8]. As there is no cure for $\mathrm{VaD}$, management of $\mathrm{VaD}$ emphasizes on the prevention of new stroke and control of vascular risk factors.

\section{Dementia and Chinese medicine}

According to Chinese medicine theory, there is no distinction between $\mathrm{AD}$ and $\mathrm{VaD}$. Dementia is caused by (1) deficiency of vital energy of the Kidney (Shen), Marrow (Sui), Heart (Xin) and Spleen (Pi) and (2) stagnation of Blood (Xie) and/or Phlegm (Tan). Thus, herbs used for dementia are not specific for the nervous system but tend to be multi-functional [9].

\section{Standardization of dementia subtype classification and research guidelines}

Guideline for Chinese Medicine Diagnosis, Classification and Clinical Research of Senile Dementia was published in 1990. The guideline classified dementia into six subtypes according to the CM theory: (1) the Bone Marrow (Gusui) deficiency syndrome, (2) the Liver (Gan) and Kidney (Shen) Yin deficiency syndrome, (3) the Spleen $(P i)$ and Kidney (Shen) Yang deficiency syndrome, (4) the Qi stagnation and Blood (Xie) stasis syndrome, (5) the Phlegm Turbid (Tan Zhuo) blocking Orifice (Qing Qiao) syndrome, and (6) the Heart (Xin) and Liver Fire (Gan Huo) syndrome [10]. Since then, clinical studies on dementia in China have been based on this guideline [11]. More recently, the Guideline Principles for Clinical Research on New Chinese Medicine (trial version) [3] provides more detailed description on the diagnostic criteria and describes the severity of disease subtypes quantitatively. The Mini-Mental State Examination (MMSE) score has also been introduced as the main reference index [3]. Criteria for the Diagnosis, Differentiation of Syndrome and Evaluation of Efficacy of Vascular Dementia for Research Studies were published on 2002, emphasizing that the diagnosis of $\mathrm{VaD}$ must meet the NINDS-AIREN criteria (developed by the National Institute of Neurological Disorders and Stroke (NINDS) and the Association Internationale pour la Recherche et l'Enseignement en Neurosciences (AIREN)) and that the differentiation of syndromes in Chinese medicine should be based on the scale for the differentiation of syndromes of vascular dementia (SDSVD) published in 2000 [12]. It classifies $\mathrm{VaD}$ in 7 syndromes according to CM diagnosis: (1) the Kidney Essence (Shen Jing) deficiency syndrome, (2) the Phlegm Turbid (Tan Zhuo) blocking Orifice (Qing Qiao) syndrome, (3) the vessels obstructed by Blood Stasis (Xie Yu) syndrome, (4) the brain aggressed by Liver's (Gan) Yang syndrome, (5) the Heat $(R e)$ and Toxin $(D u)$ accumulation syndrome, (6) the Qi and Blood (Xie) deficiency syndrome, and (7) the constipation and toxin in intestines syndrome. SDSVD employs a detailed scoring system to assist syndrome differentiation and diagnosis [13].

\section{Chinese medicine approaches based on the etiology of dementia}

Chinese medicine theory considers dementia to be a more holistic and integrated approach, rather than a problem in just one organ. Dementia is complex and may involve multiple causes. During progression of dementia, the significant of different pathological factors may also change. In Chinese medicine, it is believed that the disease is highly correlated to the abnormal functions of other organs including the Kidney (Shen), Liver (Gan), Heart (Xin) and Spleen (Pi), although the pathological site of dementia is in the brain. For example, dementia patients who initially have Kidney (Shen) deficiency may also develop stagnation of Blood (Xie) and Phlegm (Tan) leading to dementia. All these clinical experiences, stagnation of blood and kidney deficiency, become two important concepts in Chinese medicine to explain the origin of sickness leading to dementia [14-16].

\section{Chinese medicine studies on the prevalence and distribution} of dementia subtypes

In order to integrate Chinese medicine diagnosis, some researchers investigated the prevalence and distribution of dementia subtypes. Wang et al. found that deficiency of Qi, Blood (Xie), Essence (Jing) was present in most dementia cases and that Heart (Xin) and Kidney (Shen) were most commonly afflicted by the condition [17]. Yang et al. found that stagnation of Blood (Xie) and Phlegm (Tan) were frequently present in moderate and severe AD cases [18]. While these data are not diagnostic criteria, they provide important information for the prevention of pathological progression in dementia.

\section{Chinese medicine research on treatment of dementia}

Nowadays, the development of Chinese herbal medicine mainly adopted three approaches, which include the single molecule approach, standardized extracted approach and fixed herbal formula approach. In the following sections, we will discuss the recent development of each approach. It is beyond the scope of our report to review the pharmacological effects of all medicinal herbs for dementia treatment in detail. In fact, our aim is to use several representative examples to illustrate the advantages and disadvantages of each approach.

\section{Single molecules from a single herb}

Huperzine A Huperzine A is an alkaloid isolated from Huperzia serrata (Qiancengta) which is documented in Chinese medicine literature as an anti-inflammatory herb for relieving pain and alleviating swelling after trauma. According to the Chinese medicine theory, Huperzia serrata helps removing Heat $(R e)$ and has detoxification effects. Huperzine A is widely used in China to treat AD. Clinical trials demonstrated that huperzine A significantly improved cognitive functions 
in AD patients [19] and its potential therapeutic effects for $\mathrm{VaD}$ [20]. While evidence for the use of huperzine A to treat $\mathrm{AD}$ was insufficient, a recent Cochrane systematic review reported no obvious adverse effects in $A D$ patients treated with huperzine A [21].

The anti-AChE activity of huperzine $\mathrm{A}$ is the basis for its use to treat dementia patients [22]. Animal studies found that huperzine A was comparable to donepezil and rivastigmine in terms of anti-AChE activity [23]. In vivo studies showed that huperzine A affected amyloid precursor protein processing to reduce the formation of $A \beta$ peptides [24]. Other studies also showed that huperzine A attenuated apoptosis in neurons treated with $A \beta$ peptides [25]; and huperzine A elicited anti-oxidative effects which allowed it to protect neurons against hydrogen peroxide and $A \beta$-induced oxidative damages $[26,27]$. Huperzine A was found to improve mitochondrial functions in neurons and reduce the level of reactive oxygen species in neurons exposed to $\mathrm{A} \beta$ peptides [28].

Pros and cons of the single molecule approach Huperzine A is a chemical drug derived from Chinese medicine as a chemical library [29]. The discovery of huperzine $\mathrm{A}$ as an anti-AChE inhibitor was based on the observation that the administration of Huperzia serrata extract induced cholinergic stimulation in schizophrenic patients [30]. As the quality and bioavailability of a herb can be affected by the cultivation environment and harvesting season [31], single molecule approach eliminates or minimizes this variation.

However, this approach may also generate problems. Extraction of active ingredients is often not a simple task. Interactions of ingredients during preparation procedure are essential to the therapy. Moreover, evidence shows that single component extracted from plants is less potent than crude extract [32]. Researchers often do not use any Chinese medicine theory as the basis for their investigation when studying these compounds. Therefore, some Chinese medicine experts are skeptical about the approach [33].

\section{Standardized extracts from a single herb}

Authentication of herbs Historically, herbs grown in a particular habitat are considered Daodi (genuine) [34]. Today, the good agricultural practice (GAP) promoted in China ensures the quality and consistency of a particular herb [35]. A herbal extract is considered 'standardized' if (1) the raw material (herb) is grown and collected according to the GAP; (2) the extraction follows a well-defined procedure; and (3) the chemical profiles are consistent among batches of extracts.

EGb761 EGb761 is a standardized herbal extract from the dried leafs of Ginkgo biloba, containing approximately $24 \%$ flavone glycosides (quercetin, kaempferol and isorhamnetin) and 6\% terpene lactones (ginkgolides A, B, C, J and bilobalide) [36]. Dried fruit of Ginkgo biloba is used in Chinese medicine to treat asthma and coughing. While the chemical and biological properties of individual EGb761 component have been investigated in vitro and in vivo [37-39], the standardized extract EGb761 is often used in clinical research [40]. A Cochrane systematic review did not support the use of EGb761 in dementia treatment [40]. Another report also suggested that EGb761 was not effective in reducing the incidence of AD [41]. Although clinical efficacy of EGb761 for dementia treatment is still controversial, the use of a well-defined herbal extract in clinical studies has been demonstrated.

Chinese medicine theories and anti-dementia drug research Chinese medicine theory and western pharmacology may be integrated for the development of antidementia Chinese herbal extracts. According to Chinese medicine theory, the fruit of L. barbarum (Gouqizi), which is used to tonify the Yin in our body, nourishes our Eye (Yan), Liver (Gan) and Kidney (Shen); its antiaging effects are well-documented in Chinese medicine literature [42]. In our laboratory, research of standardized $L$. barbarum extract is based on Chinese medicine concepts. Firstly, L. barbarum is chosen as the research candidate because of its unique Chinese medicine properties. Secondly, the research direction of our standardized L. barbarum extract was inspired by Chinese medicine theory. Owing to anti-aging properties, $L$. barbarum may alleviate aging-associated neurodegenerative diseases such as $\mathrm{AD}$ and $\mathrm{VaD}$ [9]. Quality control of our raw materials ensured the quality of our $L$. barbarum extract [43]. We found that L. barbarum extract attenuated $A \beta$ peptide induced neuronal apoptosis [43]. The holistic concept in Chinese medicine inspired us to study the effects of the extract on other dementia related pathological and risk factors. We then discovered that $L$. barbarum extract protected neurons against glutamate toxicity, suggesting that it might slow down dementia progression [44]. We also demonstrated that L. barbarum extract protected neurons against homocysteine toxicity where hyperhomocysteinaemia is a risk factor for AD [45].

\section{Herbal formulations}

Yokukansan Yokukansan, or TJ-54, is a Kampo herbal remedy originating from the Chinese herbal formula Yigan San developed in the Song Dynasty for the treatment of Liver (Gan) dysfunction-induced agitation and restlessness in children. Yigan San consists of seven herbs, namely Angelica acutiloba (Danggui), Atractylodes lancea (Baishu), Bupleurum falcatum (Chaihu), Poria cocos (Fuling), Cnidium officinale (Chuanxiong), Uncaria rhynchophylla (Gouteng) and Glycyrrhiza uralensis (Gancao) at a ratio of 3:4:2:4:3:3:1.5. This composition is also used in Yokukansan [46]. Since this remedy is used for the treatment of psychiatric disorder, 
the possible therapeutic effects on dementia symptoms are under investigation.

Both clinical and preclinical studies on Yokukansan support its use in dementia treatment. A randomized, observer-blind, controlled trial found that a 4-week Yigan San treatment improved the behavioral and psychological symptoms of dementia (BPSD) [47] which includes aggression, agitation, screaming, wandering, hallucinations and delusions. These symptoms develop in $20-80 \%$ of dementia patients at different stages [48]. Yigan San reduced cholinesterase inhibitor-resistant visual hallucination in a small group of patients of dementia with Lewy bodies [49]. Positive effects of Yokukansan on sleep disturbance in dementia patients were also reported [50]. A randomized cross-over study (subjects receiving active treatment or placebo in different stages of the trial) found that Yokukansan significantly improved the BPSD in AD patients but had no effects on their cognitive functions as demonstrated by the MMSE score. Effects of Yokukansan could persist for one month and was well-tolerated [51]. Other studies also found that Yokukansan was safe and effective in treating BPSD in AD and even PD patients $[52,53]$. Yokukansan might modulate the glutamatergic neurotransmitter system; hence protecting neurons against excitotoxicity $[54,55]$. Yokukansan provided direct protection on neurons or through modulating the glutamate reuptake by astrocytes [56]. Yokukansan also affected the expression of serotonin receptor in the frontal cortex of mice injected with 2,5-dimethoxy-4iodoamphetamine [57].

Challenges in developing anti-dementia herbal formulations Apart from Yigan San, some other Chinese herbal formulae are effective in treating the dementia $[58,59]$. Most of these studies are single clinical trials performed on a single formula. There are few studies on action mechanisms. Moreover, few studies use Chinese medicine diagnostic criteria. In other words, the effects of a definite formula were tested on patients regardless of their dementia subtype in Chinese medicine diagnosis. As exemplified in a clinical trial for respiratory diseases, it should be feasible to incorporate Chinese medicine diagnosis in the clinical trials for dementia [60].

\section{Conclusion}

While Chinese herbal medicine is considered a big chemical library, potential drugs of single molecules have been developed for the treatment of $\mathrm{AD}$ and $\mathrm{VaD}$ but Chinese medicine concepts have not been fully incorporated for new drug development in Chinese medicine. As Chinese medicine aims to restore harmony of the whole body rather than only target the brain in treating encephalopathy [61], further research into experimental and clinical sciences should be conducted to explain how Chinese medicine can treat and prevent AD and $\mathrm{VaD}$.

\section{Abbreviations}

AD: Alzheimer's disease; Aß: beta-amyloid; AChE: acetylcholinesterase; BPSD: behavioral and psychological symptoms of dementia; MMSE: Mini-Mental State Examination; NINDS-AIREN: National Institute of Neurological Disorders and Stroke (NINDS) and the Association Internationale pour la Recherche et I'Enseignement en Neurosciences (AIREN); SDSVD: scale for the differentiation of syndromes of vascular dementia; VaD: vascular dementia.

\section{Acknowledgements}

The work of Chinese Medicine research in this laboratory is partly supported by Azalea (1972) Foundation, The University of Hong Kong (HKU) Alzheimer's Disease Research Network under Strategy Research Theme on Healthy Aging, HKU Strategic Research Theme on Drug Discovery, Area of Excellent in Institute of Molecular Technology for Drug Discovery and Synthesis, and HKU Technology Transfer Seed Funding.

\section{Author details}

${ }^{1}$ Laboratory of Neurodegenerative Diseases, Department of Anatomy, The University of Hong Kong Pokfulam, Hong Kong SAR, China. ${ }^{2}$ Research Centre of Heart, Brain, Hormone and Healthy Aging, LKS Faculty of Medicine, The University of Hong Kong Pokfulam, Hong Kong SAR, China. ${ }^{3}$ State Key Laboratory of Brain and Cognitive Sciences, The University of Hong Kong Pokfulam, Hong Kong SAR, China.

\section{Authors' contributions}

KFS and RCCC contributed the main theme ideas. YSH wrote the manuscript. All authors read and approved the final version of the manuscript.

\section{Competing interests}

The authors declare that they have no competing interests.

Received: 8 December 2010 Accepted: 22 April 2011

Published: 22 April 2011

\section{References}

1. Alzheimer's Association: Alzheimer's disease facts and figures. Alzheimers Dement 2010, 6:158-194.

2. Ferri CP, Prince M, Brayne C, Brodaty H, Fratiglioni L, Ganguli M, Hall K, Hasegawa K, Hendrie H, Huang Y, Jorm A, Mathers C, Menezes PR, Rimmer E, Scazufca M: Global prevalence of dementia: a Delphi consensus study. Lancet 2005, 366:2112-2117.

3. Song $Y$, Wang J: Overview of Chinese research on senile dementia in mainland China. Ageing Res Rev 2010, , Suppl 1: S6-12.

4. Querfurth HW, LaFerla FM: Alzheimer's disease. N Engl J Med 2010, 362:329-344.

5. Iqbal K, Grundke-lqbal I: Alzheimer neurofibrillary degeneration: significance, etiopathogenesis, therapeutics and prevention. J Cell Mol Med 2008, 12:38-55.

6. Mayeux R: Clinical practice. Early Alzheimer's disease. N Engl J Med 2010, 362:2194-2201.

7. Gorelick PB: Risk factors for vascular dementia and Alzheimer disease. Stroke 2004, 35(Suppl 1):2620-2622.

8. Lindsay J, Hebert R, Rockwood K: The Canadian study of health and aging: risk factors for vascular dementia. Stroke 1997, 28:526-530.

9. Ho YS, So KF, Chang RCC: Anti-aging herbal medicine-how and why can they be used in aging-associated neurodegenerative diseases? Ageing Res Rev 2010, 9:354-362.

10. Fu RJ: Guidelines for TCM diagnosis, classification and clinical research of senile dementia. J Tradit Chin Med 1991, 2:56-57.

11. Fu RJ, Luo SW: Review on clinical research of senile dementia treated by TCM. J Beijing University of TCM 1994, 3:146-152.

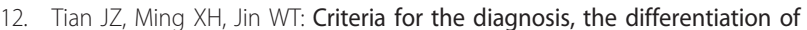
syndrome and the evaluation of efficacy of vascular dementia for research studies. Chin J Gerontol 2002, 5:329-331. 
13. Tian JZ, Han XM, Yue JM, Yang CZ, Yang HM, Zou YH, Liu X: The scale for the differentiation of syndromes of vascular dementia (SDSVD). J Beijing University TCM 2000, 23:16-24

14. Dong HT, Jin YG: A discussion on the etiology and pathogenesis of senile dementia due to stagnation of blood-stasis in the orifice. Nanjing University of TCM 2003, 35:3-4.

15. Zhang G, Wang FW: Recent progress in Chinese medicine clinical research on vascular dementia. Lishizhen Med Mater Med Res 2010, 3:769-771.

16. Yuen DP, Qiu XF, Wang P, Zhou AF: Deficiency of kidney and febility of marrow, blockage of brain vollateral: basic pathogenesis of Alzheimer's disease (AD). Zhonghua Zhongyiyao Zazhi 2008, 8:732-734.

17. Wang $X Y$, Liu CP, Chen YW: Recent progress in TCM research on Alzheimer's disease pathogenesis. Neimengguz Zhong Yi Yao 2008, 12:62-64.

18. Yang BC, Liu RR, Xue RH, Ye SL: Research on TCM pattern identification in dementia of the Alzheimer type. Shanghai J Tradit Chin Med 2000, 4:12-14.

19. Wang BS, Wang H, Wei ZH, Song YY, Zhang L, Chen HZ: Efficacy and safety of natural acetylcholinesterase inhibitor huperzine $A$ in the treatment of Alzheimer's disease: an updated meta-analysis. J Neural Transm 2009, 116:457-465.

20. Wang R, Yan H, Tang XC: Progress in studies of huperzine A, a natural cholinesterase inhibitor from Chinese herbal medicine. Acta Pharmacol $\sin 2006,27: 1-26$.

21. Li J, Wu HM, Zhou RL, Liu GJ, Dong BR: Huperzine A for Alzheimer's disease. Cochrane Database Syst Rev 2008, , 2: CD005592.

22. Ma X, Tan C, Zhu D, Gang DR, Xiao P: Huperzine A from Huperzia species-an ethnopharmacolgical review. J Ethnopharmacol 2007, 113:15-34.

23. Liang YQ, Tang XC: Comparative studies of huperzine A, donepezil, and rivastigmine on brain acetylcholine, dopamine, norepinephrine, and 5hydroxytryptamine levels in freely-moving rats. Acta Pharmacol Sin 2006 27:1127-1136.

24. Zhang HY, Yan H, Tang XC: Huperzine A enhances the level of secretory amyloid precursor protein and protein kinase $C$-alpha in intracerebroventricular beta-amyloid-(1-40) infused rats and human embryonic kidney 293 Swedish mutant cells. Neurosci Lett 2004 360:21-24.

25. Xiao $X Q$, Zhang $H Y$, Tang $X C$ : Huperzine $A$ attenuates amyloid betapeptide fragment 25-35-induced apoptosis in rat cortical neurons via inhibiting reactive oxygen species formation and caspase- 3 activation. J Neurosci Res 2002, 67:30-36.

26. Wang $R$, Xiao $X Q$, Tang XC: Huperzine $A$ attenuates hydrogen peroxideinduced apoptosis by regulating expression of apoptosis-related genes in rat PC12 cells. Neuroreport 2001, 12:2629-2634.

27. Xiao $X Q$, Wang $R$, Tang XC: Huperzine $A$ and tacrine attenuate betaamyloid peptide-induced oxidative injury. J Neurosci Res 2000, 61:564-569.

28. Gao X, Tang XC: Huperzine A attenuates mitochondrial dysfunction in beta-amyloid-treated PC12 cells by reducing oxygen free radicals accumulation and improving mitochondrial energy metabolism. J Neurosci Res 2006, 83:1048-1057.

29. Pan SY, Chen SB, Dong HG, Yu ZL, Dong JC, Long ZX, Fong WF, Han YF, Ko KM: New perspectives on Chinese herbal medicine (Zhong-Yao) research and development. Evid Based Complement Alternat Med 2010, 2011:403709.

30. Wang Y, Huang LQ, Tang XC, Zhang HY: Retrospect and prospect of active principles from Chinese herbs in the treatment of dementia. Acta Pharmacol Sin 2010, 31:649-664.

31. Anekonda TS, Reddy PH: Can herbs provide a new generation of drugs for treating Alzheimer's disease? Brain Res Rev 2005, 50:361-376.

32. Luo Q, Cai Y, Yan J, Sun M, Corke H: Hypoglycemic and hypolipidemic effects and antioxidant activity of fruit extracts from Lycium barbarum. Life Sci 2004, 76:137-149.

33. Normile D: Asian medicine. The new face of traditional Chinese medicine. Science 2003, 299:188-190.

34. Leung PC, Cheng KF: Good agricultural practice (GAP) -Does it ensure a perfect supply of medicinal herbs for research and drug development? Int J Appl Res Nat Prod 2008, 1:1-8.

35. Zhang B, Peng Y, Zhang Z, Liu H, Qi Y, Liu S, Xiao P: GAP production of TCM herbs in China. Planta Med 2010, 76:1948-1955.
36. Clostre F: Ginkgo biloba extract (EGb 761). State of knowledge in the dawn of the year 2000. Ann Pharm Fr 1999, 57(Suppl 1):1S8-88.

37. Chen WD, Liang Y, Xie L, Lu T, Liu XD, Wang GJ: Pharmacokinetics of the ginkgo $B$ following intravenous administration of ginkgo $B$ emulsion in rats. Biol Pharm Bull 2007, 30:1-5.

38. Lee TF, Chen CF, Wang LC: Effect of ginkgolides on beta-amyloidsuppressed acetylocholine release from rat hippocampal slices. Phytother Res 2004, 18:556-560.

39. Zhou $\sqcup$, Zhu $X Z$ : Reactive oxygen species-induced apoptosis in PC12 cells and protective effect of bilobalide. J Pharmacol Exp Ther 2000, 293:982-988.

40. Birks J, Grimley EJ: Ginkgo biloba for cognitive impairment and dementia. Cochrane Database Syst Rev 2009, , 1: CD003120.

41. Dekosky ST, Williamson JD, Fitzpatrick AL, Kronmal RA, Ives DG, Saxton JA, Lopez OL, Burke G, Carlson MC, Fried LP, Kuller LH, Robbins JA, Tracy RP, Woolard NF, Dunn L, Snitz BE, Nahin RL, Furberg CD: Ginkgo biloba for prevention of dementia: a randomized controlled trial. JAMA 2008, 300:2253-2262.

42. Chang RCC, So KF: Use of Anti-aging Herbal Medicine, Lycium barbarum, Against Aging-associated Diseases. What Do We Know So Far? Cell Mol Neurobiol 2007, 28:643-652.

43. Yu MS, Leung SK, Lai SW, Che CM, Zee SY, So KF, Yuen WH, Chang RCC: Neuroprotective effects of anti-aging oriental medicine Lycium barbarum against beta-amyloid peptide neurotoxicity. Exp Gerontol 2005, 40:716-727.

44. Ho YS, Yu MS, Yik SY, So KF, Yuen WH, Chang RCC: Polysaccharides from wolfberry antagonizes glutamate excitotoxicity in rat cortical neurons. Cell Mol Neurobiol 2009, 29:1233-1244.

45. Ho YS, Yu MS, Yang XF, So KF, Yuen WH, Chang RCC: Neuroprotective effects of polysaccharides from wolfberry, the fruits of Lycium barbarum, against homocysteine-induced toxicity in rat cortical neurons. J Alzheimers Dis 2010, 19:813-827.

46. Mizoguchi K, Tanaka Y, Tabira T: Anxiolytic effect of a herbal medicine, yokukansan, in aged rats: Involvement of serotonergic and dopaminergic transmissions in the prefrontal cortex. J Ethnopharmacol 2010, 127:70-76.

47. Iwasaki K, Satoh-Nakagawa T, Maruyama M, Monma Y, Nemoto M, Tomita N, Tanji H, Fujiwara H, Seki T, Fujii M, Arai H, Sasaki H: A randomized, observer-blind, controlled trial of the traditional Chinese medicine Yi-Gan San for improvement of behavioral and psychological symptoms and activities of daily living in dementia patients. J Clin Psychiatry 2005, 66:248-252.

48. Lawlor BA: Behavioral and psychological symptoms in dementia: the role of atypical antipsychotics. J Clin Psychiatry 2004, 65(Suppl 11):5-10.

49. Iwasaki K, Maruyama M, Tomita N, Furukawa K, Nemoto M, Fujiwara H, Seki T, Fujii M, Kodama M, Arai H: Effects of the traditional Chinese herbal medicine Yi-Gan San for cholinesterase inhibitor-resistant visual hallucinations and neuropsychiatric symptoms in patients with dementia with Lewy bodies. J Clin Psychiatry 2005, 66:1612-1613.

50. Shinno $H$, Inami $Y$, Inagaki T, Nakamura $Y$, Horiguchi J: Effect of Yi-Gan San on psychiatric symptoms and sleep structure at patients with behavioral and psychological symptoms of dementia. Prog Neuropsychopharmacol Biol Psychiatry 2008, 32:881-885.

51. Mizukami K, Asada T, Kinoshita T, Tanaka K, Sonohara K, Nakai R, Yamaguchi K, Hanyu H, Kanaya K, Takao T, Okada M, Kudo S, Kotoku H, Iwakiri M, Kurita H, Miyamura T, Kawasaki Y, Omori K, Shiozaki K, Odawara T, Suzuki T, Yamada S, Nakamura Y, Toba K: A randomized cross-over study of a traditional Japanese medicine (kampo), yokukansan, in the treatment of the behavioural and psychological symptoms of dementia. Int J Neuropsychopharmacol 2009, 12:191-199.

52. Kawanabe T, Yoritaka A, Shimura H, Oizumi H, Tanaka S, Hattori N: Successful treatment with Yokukansan for behavioral and psychological symptoms of Parkinsonian dementia. Prog Neuropsychopharmacol Biol Psychiatry 2010, 34:284-287.

53. Okahara $K$, Ishida $Y$, Hayashi $Y$, Inoue T, Tsuruta $K$, Takeuchi $K$, Yoshimuta $H$, Kiue K, Ninomiya Y, Kawano J, Yoshida K, Noda S, Tomita S, Fujimoto M, Hosomi J, Mitsuyama Y: Effects of Yokukansan on behavioral and psychological symptoms of dementia in regular treatment for Alzheimer's disease. Prog Neuropsychopharmacol Biol Psychiatry 2010, 34:532-536. 
54. Takeda A, Itoh H, Tamano H, Yuzurihara M, Oku N: Suppressive effect of Yokukansan on excessive release of glutamate and aspartate in the hippocampus of zinc-deficient rats. Nutr Neurosci 2008, 11:41-46.

55. Takeda A, Tamano H, Itoh H, Oku N: Attenuation of abnormal glutamate release in zinc deficiency by zinc and Yokukansan. Neurochem Int 2008, 53:230-235.

56. Kawakami Z, Kanno H, Ueki T, Terawaki K, Tabuchi M, Ikarashi Y, Kase Y: Neuroprotective effects of yokukansan, a traditional Japanese medicine, on glutamate-mediated excitotoxicity in cultured cells. Neuroscience 2009, 159:1397-1407.

57. Egashira N, Iwasaki K, Ishibashi A, Hayakawa K, Okuno R, Abe M, Uchida N, Mishima K, Takasaki K, Nishimura R, Oishi R, Fujiwara M: Repeated administration of Yokukansan inhibits DOI-induced head-twitch response and decreases expression of 5-hydroxytryptamine (5-HT)2A receptors in the prefrontal cortex. Prog Neuropsychopharmacol Biol Psychiatry 2008, 32:1516-1520.

58. Zhu AH, Tian JZ, Zhong J, Yang CZ, Shi J, Yin JX: A clinical study on a randomized, double-blind control of Chinese medicine granules in treatment of vascular dementia. Zhongguo Zhong Yao Za Zhi 2006, 31:1722-1725.

59. Du GY, Zhu XC, Zhao JJ, Wang J, Tian JZ, Liu XF, Zhi HP, Zhao Y, Cao CY, Cui HF, Wang XR, Zhang CY: Clinical study of effect of tianzhi granule on senile vascular dementia. Zhongguo Zhong Yao Za Zhi 2003, 28:73-77.

60. Lam CLK, Wong W, Fong DY: Chinese herbal medicine in the treatment of acute upper respiratory tract infection: a randomised, double blind, placebo-controlled clinical trial. Hong Kong Med J 2009, 15(Suppl 6):30-34.

61. Liu W, Zhang GL, Wang XL: Application and prospects of fan-sui relation in TCM in preventing and treating encephalopathy. Zonghua Zhongyiyao Za Zhi 2010, 25:993-996.

doi:10.1186/1749-8546-6-15

Cite this article as: Ho et al:: Drug discovery from Chinese medicine against neurodegeneration in Alzheimer's and vascular dementia. Chinese Medicine 2011 6:15.

\section{Submit your next manuscript to BioMed Central and take full advantage of:}

- Convenient online submission

- Thorough peer review

- No space constraints or color figure charges

- Immediate publication on acceptance

- Inclusion in PubMed, CAS, Scopus and Google Scholar

- Research which is freely available for redistribution

Submit your manuscript at www.biomedcentral.com/submit
Biomed Central 